\title{
Descompresión anterior y fijación con cajas autobloqueantes en columna cervical
}

\author{
Anterior cervical decompression and fusion with PEEK cages

\begin{abstract}
Anabel Euán Gutiérrez, ${ }^{*}$ Carla Lisette García Ramos, ${ }^{~ L u i s ~ M i g u e l ~ R o s a l e s ~ O l i v a r e z, ~}{ }^{*}$
Armando Alpizar Aguirre, ${ }^{\ddagger}$ Alejandro Reyes Sánchez*

*Médico Cirujano, Especialista en Traumatología y Ortopedia; ${ }^{\ddagger}$ Médico Cirujano, Especialista en Neurocirugía.

Alta Especialidad en Cirugía de Columna, Instituto Nacional de Rehabilitación "Luis Guillermo Ibarra Ibarra». Ciudad de
\end{abstract} \\ México, México.
}

\begin{abstract}
Resumen
Introducción: La mielopatía cervical se define como el daño de la médula espinal debido a cambios degenerativos de la columna cervical. Además, es la causa más común de disfunción medular en pacientes mayores de 55 años, por lo tanto, el entendimiento de la patología y su manejo oportuno es importante. En esta revisión nos enfocamos en el manejo quirúrgico y los cambios del mismo desde su inicio hasta el manejo actual, incluyendo nuestra experiencia en el Instituto Nacional de Rehabilitación. Material y métodos: Se realizó una extensa revisión de la literatura publicada con enfoque principal en el manejo quirúrgico actual de la patología. Resultados: La presentación clínica y la historia natural de la enfermedad es variable; sin embargo, debido al deterioro neurológico que presentan estos pacientes, el tratamiento quirúrgico oportuno es esencial. La discectomía y fusión cervical anterior (DFCA) es el procedimiento quirúrgico habitual para conseguir la descompresión de los elementos neurales de la columna cervical. El tipo de implantes que más se utilizan son las cajas de PEEK (polyether ether ketone) obteniendo resultados clínicos y radiográficos significativos; sin embargo, aún se debate sobre el número de niveles. Conclusiones: La DFCA multinivel con cajas de PEEK es actualmente una de las técnicas más utilizadas con resultados clínicos y radiográficos significativos, los cuales se traducen en una mejor calidad de vida para los pacientes; sin embargo, aún se deben realizar más estudios que nos permitan mejorar esta técnica quirúrgica y evitar posibles complicaciones.
\end{abstract}

Palabras clave: Mielopatía cervical, discectomía y fusión cervical anterior, columna cervical, discectomía cervical multinivel, perfil cero, cajas cervicales.

\begin{abstract}
Introduction: Cervical myelopathy is defined as damage to the spinal cord due to degenerative changes in the cervical spine. In addition, is the most common cause of spinal cord dysfunction in patients older than 55 years, therefore, its understanding and opportune management is important. In this revision we focus on its surgical management and the changes it has presented since the beginning until its current management, including our experience at the National Rehabilitation Institute. Material and methods: A long review of the published literature was carried out with a main focus on its current surgical management. Results: The clinical presentation and natural history of the disease is variable, however, due to the neurological impairment that these patients present, the need to perform a promptly surgical management is essential. Anterior cervical discectomy and fusion (ACDF) is the usual surgical procedure to achieve decompression of the neural elements of the cervical spine, the type of implants usually used are PEEK cages, reporting significant clinical and radiographic results, however, the number of levels is still debated. Conclusions: The multilevel ACDF with PEEK cages is currently one of the most used techniques with significant clinical and radiographic results, which evolves into a better quality of life for patients, however, more studies must still be carried out to allow us to improve this technique and avoid possible complications.
\end{abstract}

Keywords: Cervical myelopathy, anterior cervical discectomy and fusion, cervical spine, multilevel cervical discectomy, zero profile, cervical cages.

Correspondencia:

Anabel Euán Gutiérrez

E-mail: anabeleuan@hotmail.com

Recibido: 10-12-2021. Aceptado: 19-12-2021.
Citar como: Euán GA, García RCL, Rosales OLM, Alpizar AA, Reyes SA. Descompresión anterior y fijación con cajas autobloqueantes en columna cervical. Orthotips. 2022; 18 (1): 49-52. https://dx.doi. org/10.35366/103732 


\section{Introducción}

La mielopatía cervical degenerativa (MCD) es una compresión de la médula espinal cervical debido a fenómenos degenerativos en la columna cervical tales como hipertrofia facetaria, osificación del ligamento longitudinal posterior, enfermedad degenerativa del disco y osteofitos. Es la causa principal de lesión de la médula espinal, de disfunción medular en pacientes mayores de 55 años y de paraparesias y cuadriparesias no traumáticas. ${ }^{1,2}$ El manejo quirúrgico temprano es el tratamiento de elección utilizado para lograr un mejor pronóstico neurológico. ${ }^{3}$ Existen múltiples técnicas quirúrgicas para el tratamiento de este padecimiento; siendo la discectomía y fusión cervical anterior (DFCA) el estándar de oro. De igual forma, existen diferentes implantes, los cuales continúan en discusión y su uso aún es controversial. Las cajas de PEEK (polyether ether ketone) se consideran biocompatibles, radiolucentes y con elasticidad similar al hueso, por lo cual es uno los materiales que más se utilizan en la actualidad. ${ }^{4-6}$

La discectomía y fusión cervical anterior (DFCA) es el procedimiento quirúrgico habitual para conseguir la descompresión de los elementos neurales de la columna cervical en distintos tipos de enfermedades tales como enfermedades degenerativas, traumatismos y tumores. ${ }^{6}$

Si nos remontamos alrededor del año 1852, encontramos que Stookey describió algunas dificultades técnicas para el abordaje posterior. Años más tarde, reconoció que el problema primario de este abordaje era la dificultad técnica para exponer y resecar estructuras comprensivas situadas en posición anterior a la
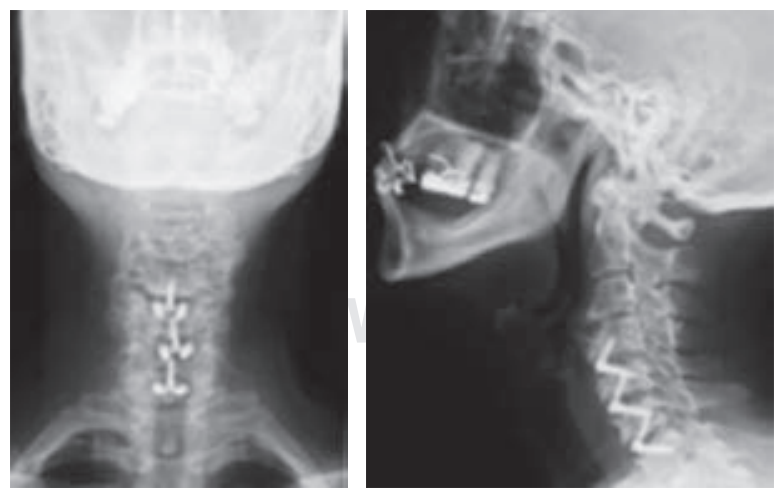

Figura 1: Radiografías anteroposterior y lateral de columna cervical postoperatoria de discectomía y fusión cervical anterior en tres niveles (C4-C5, C5-C6 y C6-C7) con cajas autobloqueantes. médula o las raíces nerviosas, por lo que el abordaje anterior se comenzó a popularizar.

Bailey y Badgley efectuaron una estabilización cervical anterior en 1952; sin embargo, la técnica apareció publicada hasta 1960. En 1955, Robinson y Smith describieron la estabilización del segmento cervical mediante un injerto. Cloward, sin conocer los trabajos previos, publicó su técnica de discectomía anterior y estabilización en 1958. Posteriormente, fueron descritas numerosas variaciones de la cirugía anterior cervical, desde las relacionadas con la configuración de los injertos hasta las relacionadas con en el material. ${ }^{7}$

\section{Material y métodos}

Se realizó una búsqueda en la base de datos de diferentes plataformas, incluyendo PubMed, OVID y Google Scholar. Los términos de búsqueda utilizados fueron: multilevel cervical discectomy, zero profile y cervical cages. Las publicaciones relacionadas fueron recolectadas para su revisión y análisis posterior.

\section{Resultados}

Esta revisión reveló que los tratamientos para la mielopatía cervical degenerativa han ido evolucionando a lo largo de la historia debido a la búsqueda continua del equilibrio entre resolver la patología de los pacientes y evitar las complicaciones.
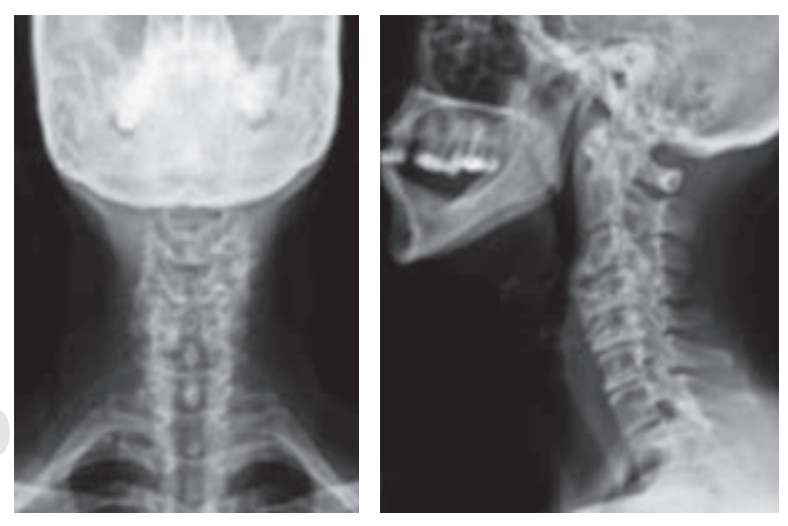

Figura 2: Radiografías anteroposterior y lateral de columna cervical donde se aprecia esclerosis subcondral, osteofitos de predominio anterior y disminución de los espacios intersomáticos multinivel que evidencian el diagnóstico de conducto cervical estrecho C4-C5, C5-C6 y C6-C7. 


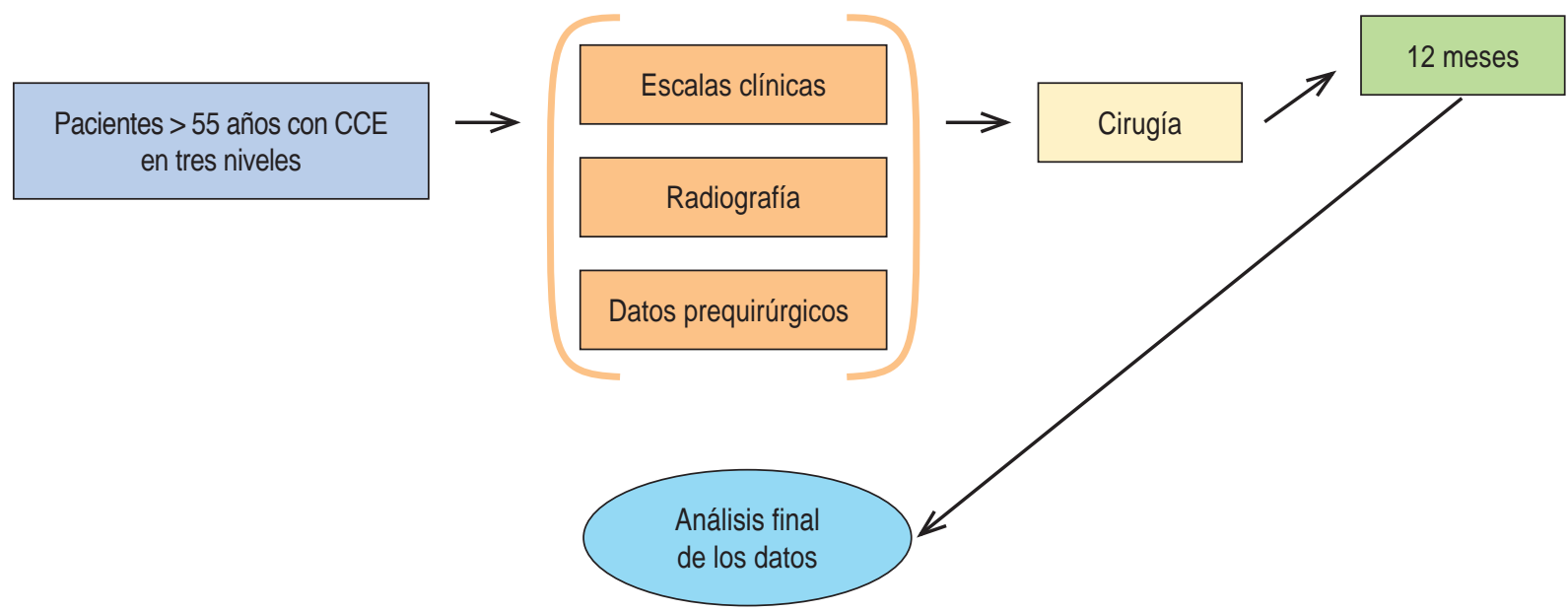

Figura 3: Se analizaron pacientes mayores de 55 años con diagnóstico de conducto cervical estrecho (CCE) en tres niveles, posteriormente se realizaron escalas clínicas (EVA, JOA, Nurick, IDC, SF-36) y mediciones radiográficas (lordosis cervical, altura del cuerpo vertebral y altura intersomática) de forma prequirúrgica. Después se efectuó la cirugía consistente en discectomía y fusión cervical anterior en tres niveles. A los 12 meses se analizaron los resultados postquirúrgicos tanto clínicos como radiográficos.

Lo ideal es que el tipo de abordaje quirúrgico se seleccione dependiendo de la presentación clínica, los hallazgos radiológicos y las comorbilidades de cada paciente. ${ }^{8}$

Las distintas técnicas utilizadas de fijación, así como los tipos de implantes empleados y el número de niveles intervenidos, se han discutido ampliamente y han evolucionado a través del tiempo con el objetivo de obtener mejores resultados y menor número de complicaciones. ${ }^{8,9}$

La discectomía cervical anterior ha sido el procedimiento que más se utiliza globalmente debido a que se realiza una incisión mínima que permite la visualización y resección directa del disco intervertebral, además de registrar menor número de complicaciones y una recuperación rápida. ${ }^{9}$

\section{Discusión}

Existen diferentes y variadas revisiones acerca del tratamiento para conseguir la descompresión de los elementos neurales de la columna cervical en patologías degenerativas. El tratamiento quirúrgico se ha enfocado en la descompresión de la médula espinal, evitar la progresión del deterioro neurológico y promover la recuperación. ${ }^{9}$

En las distintas revisiones los pacientes mostraron una mejoría tanto de la lordosis cervical como de la altura discal (Figura 1) en comparación con los resultados preoperatorios (Figura 2). Se encontraron estudios realizados con placa e injerto, mejoría en el ángulo de Cobb y en la altura intersomática $(p=0.038$ y $p=0.0004$, respectivamente). ${ }^{10}$ En estudios que evaluaron el resultado radiográfico con relación a la lordosis global no hubo diferencia estadísticamente significativa entre usar placa-injerto, placa-caja o caja de PEEK, $(p=0.003, p=0.006$ y $p=0.001$, respectivamente), lo cual nos permite efectuar un abordaje menos invasivo con resultados satisfactorios. ShanJin Wang y colaboradores demostraron en su estudio realizado en cuatro niveles con cajas de PEEK, que el ángulo de Cobb y la altura intersomática mejoraron de manera postoperatoria con una significancia clínica con un valor de $p=0.019$ y $p \leq 0.01$, respectivamente,${ }^{11}$ lo que demuestra que se puede obtener un resultado radiográfico satisfactorio independientemente de la cantidad de niveles operados.

Estudios realizados con caja-placa multinivel revelaron en la escala de JOAm (Japanese Orthopaedic Association) una diferencia significativa de $10.6 \pm 1.9$ preoperatoria a $14.4 \pm 1.8$ postoperatoria $(p \leq 0.05)$. En estudios efectuados con caja-placa multinivel demostraron que en la escala índice de discapacidad cervical (IDC) se presenta una diferencia significativa de $4.7 \pm 2.7$ preoperatoria a $13.5 \pm 3.2$ postoperatoria $(p \leq 0.05) .{ }^{12}$ Lo anterior demuestra que al realizar descompresión, discectomía y fusión cervical anterior multinivel, los pacientes presentan una mejoría funcional, valorable por medio de escalas clínicas. 


\section{Conclusiones}

La discectomía y fusión cervical anterior con cajas autobloqueantes demostró mejoría tanto en los parámetros radiográficos como en las escalas clínicas, encontrando una disminución significativa del dolor así como una mejoría en la función, la cual se traduce en una mejor calidad de vida para pacientes. Sin embargo, se deben realizar más estudios que nos permitan perfeccionar esta técnica y evitar posibles complicaciones.

\section{Protocolo de acción en el Servicio de Cirugía de Columna del Instituto Nacional de Rehabilitación «Luis Guillermo Ibarra Ibarra»}

En el Instituto Nacional de Rehabilitación «Luis Guillermo Ibarra Ibarra» (INRLGII) actualmente se realiza discectomía y fusión cervical anterior. Siendo éste un centro nacional de referencia y tomando en cuenta la bibliografía consultada, en la cual aún se debaten ciertas técnicas y material empleado dependiendo de los niveles intervenidos, se decidió dirigir un estudio en el cual se realizó descompresión anterior en tres niveles y fijación con cajas autobloqueantes en columna cervical. Se estudiaron en un periodo de cinco años (2014-2018) con el protocolo quirúrgico del servicio (Figura 3), obteniendo un total de 21 pacientes con diagnóstico de conducto cervical estrecho con o sin mielopatía, tomando en cuenta parámetros clínicos (EVA, JOA, Nurick, IDC, SF-36) y radiográficos (lordosis cervical, altura del cuerpo vertebral y altura intersomática), obteniendo resultados significativos en la muestra estudiada. En el Servicio de Cirugía de Columna del INRLGII existe en la actualidad una línea de investigación en patología degenerativa de columna cervical que busca un equilibrio entre la mejoría clínica y radiográfica que minimice las complicaciones.

\section{Referencias}

1. Marie-Hardy L, Pascal-Moussellard H. Degenerative cervical myelopathy. Rev Neurol (Paris). 2021; 177 (5): 490-497.

2. Young WF. Cervical spondylotic myelopathy: a common cause of spinal cord dysfunction in older persons. Am Fam Physician. 2000; 62 (5): 1064-1070, 1073.

3. Liu Y, Yu KY, Hu JH. Hybrid decompression technique and two-level corpectomy are effective treatments for three-level cervical spondylotic myelopathy. J Zhejiang Univ Sci B. 2009; 10 (9): 696-701.

4. Zárate-Kalfopulos B, Araos-Silva W, Reyes-Sánchez A, Rosales-Olivarez LM, Alpizar-Aguirre A, Melendez FL. Hybrid decompression and fixation technique for the treatment of multisegmental cervical spondylotic myelopathy. Int J Spine Surg. 2016; 10: 30.

5. Alpizar-Aguirre A, Estrada-Gómez JA, Zárate-Kalfopulus B, Sánchez-Bringas G, Rosales-Olivares LM, Reyes-Sánchez AA. Estudio comparativo entre placa-injerto, caja-placa y caja de PEEK en artrodesis de la columna cervical con conducto cervical estrecho. Acta Ortop Mex. 2015; 29 (1): 28-33.

6. Athanassacopoulos M, Korres DS, Pneumaticos SG. Pneumaticos. Discectomía y fusión cervical anterior a tres niveles con auto y aloinjerto. Rev Esp Cir Ortop Traumatol. 2009; 53 (6): 351-356.

7. Ferrer $\mathrm{AH}$. Tratamiento quirúrgico de la hernia de disco cervical. Técnica de Smith-Robinson. Rev Asoc Arg Ortop y Traumatol. 1998; 63 (1): 70-74

8. Kim LH, D'Souza M, Ho AL, Pendharkar AV, Sussman ES, Rezaii $P$ et al. Anterior techniques in managing cervical disc disease. Cureus. 2018; 10 (8): e3146.

9. Doria C, Mosele GR, Balsano M, Maestretti G, Caggiari G. Anterior decompression and plate fixation in treatment of cervical myelopathy: A multicentric retrospective review. Acta Orthop Traumatol Turc. 2018; 52 (3): 185-190.

10. Song KJ, Yoon SJ, Lee KB. Three- and four-level anterior cervical discectomy and fusion with a PEEK cage and plate construct. Eur Spine J. 2012; 21 (12): 2492-2497.

11. Wang SJ, Ma B, Huang YF, Pan FM, Zhao WD, Wu DS. Four-level anterior cervical discectomy and fusion for cervical spondylotic myelopathy. J Orthop Surg (Hong Kong). 2016; 24 (3): 338-343.

12. Liu $Y$, Wang $H$, Li X, Chen J, Sun H, Wang, G et al. Comparison of a zero-profile anchored spacer (ROI-C) and the polyetheretherketone (PEEK) cages with an anterior plate in anterior cervical discectomy and fusion for multilevel cervical spondylotic myelopathy. Eur Spine J. 2016; 25 (6): 1881-1890.

\section{Conflicto de intereses}

La autora y los coautores no presentan ningún tipo de conflicto de intereses con la investigación y publicación de este artículo. 